\title{
Derrame pleural y ascitis después de tratamiento con gonadotrofina coriónica humana
}

\section{Pleural effusion and ascites after human chorionic gonadotropin treatment}

\author{
Joaquín Serrano Arreba, Héctor Meijide Míguez, Javier Porteiro Sánchez, Pablo Asensio Hernández.
}

Servicio de Medicina Interna. Hospital Quirón A Coruña

\section{Resumen}

El síndrome de hiperestimulación ovárica (SHO) es una complicación frecuente entre las pacientes sometidas a tratamientos inductores de la ovulación. La característica principal del SHO es la pérdida del volumen intravascular a un tercer espacio que debe tratarse con administración de volumen evitando los diuréticos. Presentamos, a propósito de un caso, una breve revisión de los aspectos diagnósticos y terapéuticos más relevantes para el manejo de pacientes afectados por esta entidad.

Palabras clave. Hiperestimulación ovárica. Fecundación in vitro. Hiponatremia

\section{Introducción}

El síndrome de hiperestimulación ovárica (SHO) constituye una complicación iatrogénica potencialmente letal que surge como respuesta suprafisiológica ovárica tras la administración de gonadotrofina coriónica humana durante las técnicas de fecundación in vitro ${ }^{1,2,5}$. En general es una entidad autolimitada si bien en sus formas más graves puede cursar con serias complicaciones como accidentes cerebrovasculares por trombosis venosa, disfunción hepática o renal, complicaciones respiratorias, derrame pericárdico e incluso la muerte ${ }^{2-4}$.

La incidencia de este cuadro en sus formas leves varía entre un $0,6 \%$ y un $14 \%^{8}$. Casos graves, como el que aquí se

\begin{abstract}
The ovarian hyperestimulation syndrome (OHS) is a common complication that affects patients under ovulation induction treatment. The central characteristic of OHSS is the shift of fluids into the third space that should be treated with volume administration instead of diuretic treatment. We present a short review of the main diagnostic and therapeutic aspects for the management of these patients.
\end{abstract}

Key words. Ovarian hyperstimulation. In vitro fertilization. Hyponatremia

presenta, tienen una incidencia menor $(0,2 \%-5 \%)^{5-8}$. Los principales factores de riesgo para desarrollarlo son una edad menor a 30 años, un fenotipo delgado, el síndrome del ovario poliquístico y una historia previa de $\mathrm{SHO}^{1,2,8}$.

\section{Caso clínico}

Mujer de 29 años sin antecedentes médico-quirúrgicos relevantes en programa de fecundación in vitro sometida a ciclo de estimulación ovárica cinco días antes de su ingreso. Seguida en consultas externas de Ginecología refería molestias abdominales difusas, sensación nauseosa y aumento del perímetro abdominal. Negaba fiebre 0 sensación distérmica.

En la exploración física descataba semiología ascítica no a tensión

Figura 1.- Ecografía ginecológica

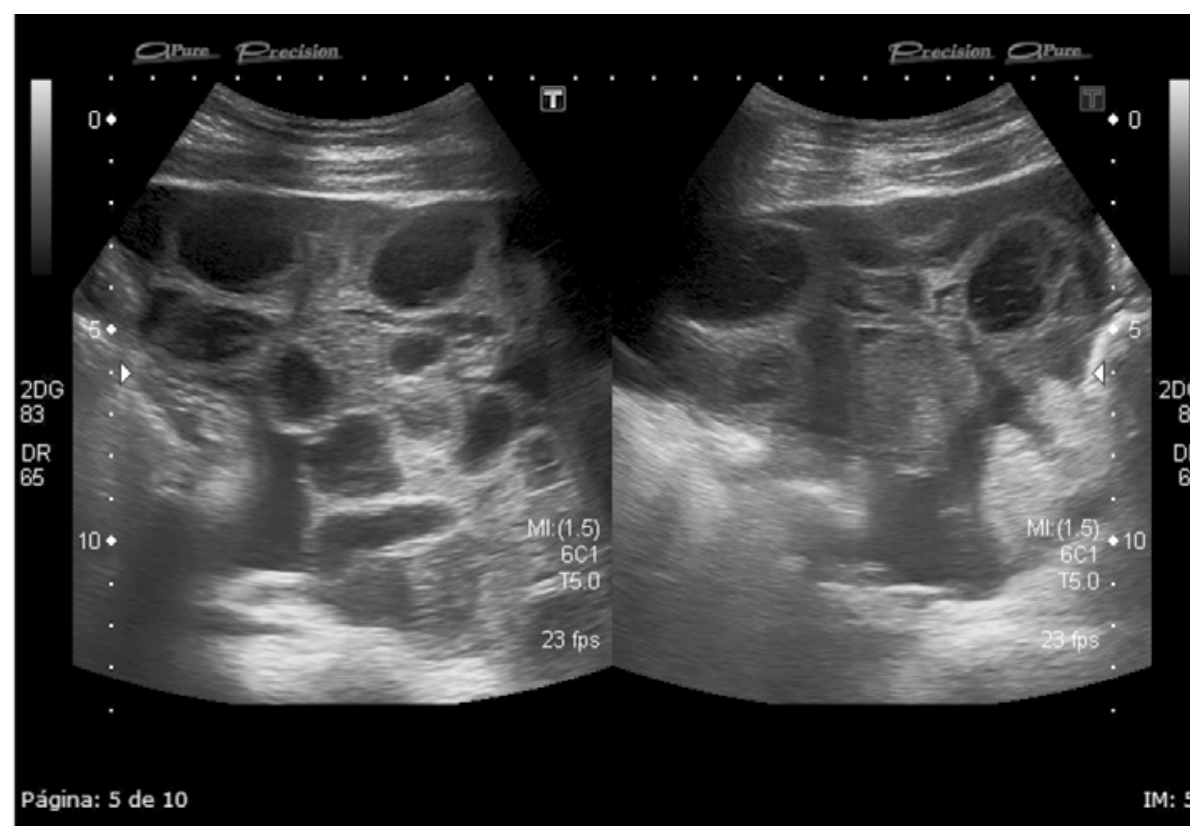


Figura 2.- TAC torácico

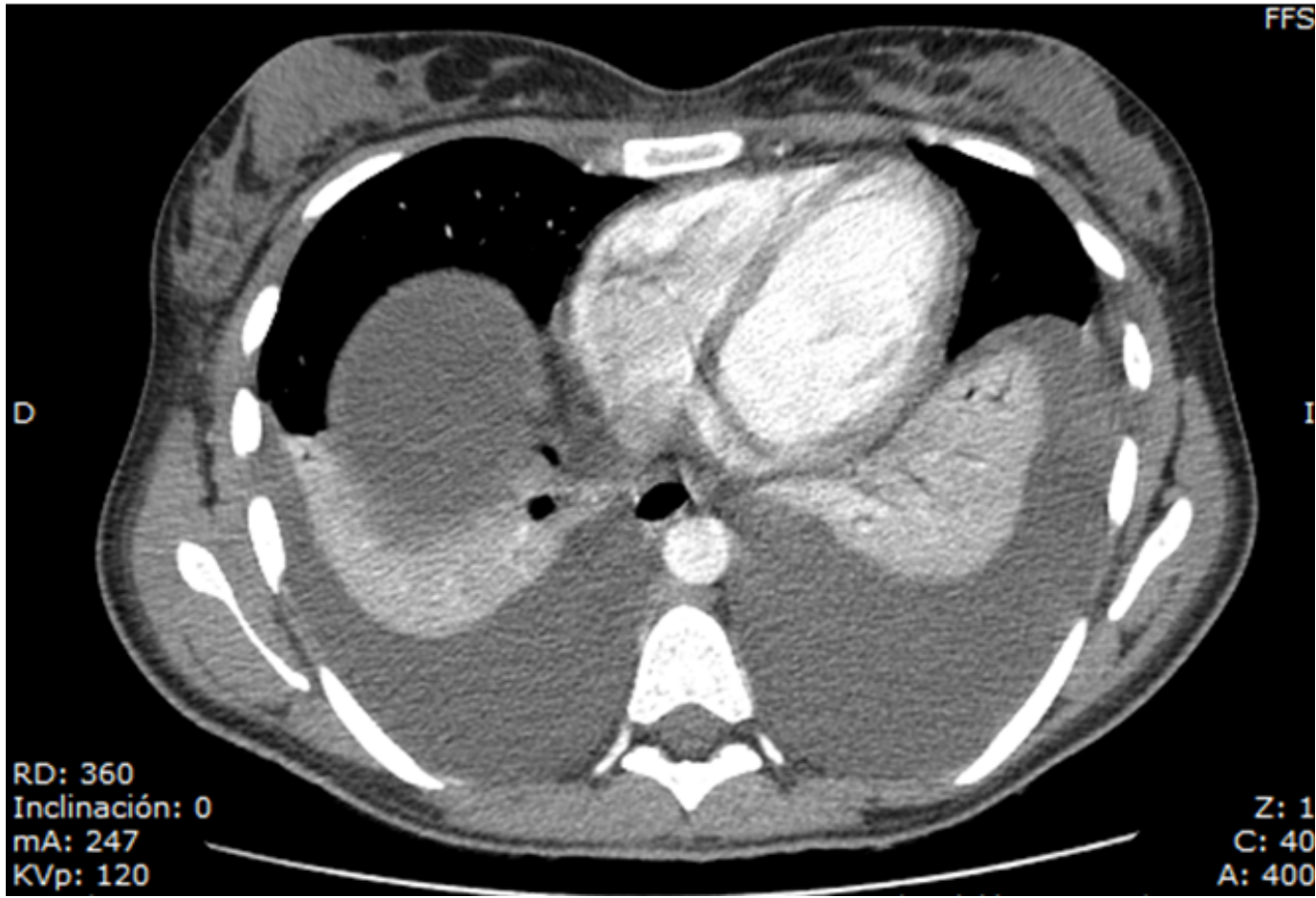

sin edema en extremidades inferiores ni ingurgitación venosa yugular. La auscultación cardiopulmonar era normal permaneciendo eupneica (14 rpm) y con TA de en torno a 110/60.

Entre los estudios complementarios destacaron 34.500 leucocitos (79\%N), TP y TTPa normales, Na de 122 mmol/L, albúmina de $2.4 \mathrm{~g} / \mathrm{L}$, siendo normal el resto de la bioquímica (glucosa, $\mathrm{K}$, urea, creatinina, LDH, Ca, bilirrubina, GGT, FA, GOT y GGT). La ecografía ginecológica mostró ascitis y tamaño ovárico de 11 cms. (Figura 1)

Con el diagnóstico de síndrome de hiperestimulación ovárica grado III ingresó en planta de hospitalización convencional. Se decidió iniciar suero fisiológico al 0,9\% y expansores de plasma (albúmina iv) con el fin de mantener un adecuado volumen intravascular y mantener así una buena perfusión renal. Dado el elevado riesgo trombótico que presentan estos pacientes se iniciaron heparinas de bajo peso molecular a dosis profilácticas.

24 horas después de su ingreso comenzó con disnea intensa y dolor pleurítico en costado izquierdo. En la exploración se objetiva taquipnea a 28-30 rpm con saturación de 02 basal del 90\% e hipofonesis en base pulmonar izquierda. Presentaba semiología ascítica no a tensión. No se objetiva aumento de la presión venosa central, signos de trombosis venosa profunda ni edema en extremidades inferiores. Los dímeros D fueron de $4200 \mathrm{ng} / \mathrm{ml}$. La radiografía de tórax confirmó derrame pleural izquierdo. Se realizó AngioTAC y TAC torácico que descartó datos de tromboembolismo pulmonar y mostró derrame pleural bilateral de predominio izquierdo y signos de edema alveolar (Figura 2).

Se procedió al traslado a UCI para vigilancia estrecha. No precisó ventilación mecánica. Se realizó paracentesis evacuadora guiada por ecografía con drenaje de 4 litros y reposición de albúmina adicional, con mejoría parcial. Pocas horas más tarde se realizó toracentesis evacuadora de 1 litro con mejoría definitiva. El cuadro se resolvió en 7 días y fue dada de alta sin secuelas.

\section{Discusión}

La patogenia del síndrome de hiperestimulación ovárica no se conoce en profundidad aunque parece que la administración exógena de hCG produciría el reclutamiento de folículos antrales, que se estimularían de manera suprafisiológica hasta producir una cantidad excesiva de factor vascular de crecimiento endotelial y causar neoangiogénesis y aumento de permeabilidad vascular1,2,9. Esto condicionaría la salida del fluido intravascular y del factor vascular del crecimiento endotelial hacia el tercer espacio, lo que causaría daño en el lecho vascular, hipovolemia, hemoconcentración, edema y ascitis, junto con fenómenos tromboembólicos secundarios a hemoconcentración 0 a alteraciones de la coagulación ${ }^{8}$.

La pérdida del volumen intravascular hacia un tercer espacio condiciona en estas pacientes una marcada hipovolemia efectiva e hipoperfusión renal secundaria. Como consecuencia se activan mecanismos compensadores (sistema nervioso simpático, sistema renina-angiotensina-aldosterona y aumento de la liberación de la hormona antidiurética) que provocan aumento en la absorción renal de sodio y agua $\left(\mathrm{H}_{2} \mathrm{O}>\mathrm{Na}\right)$ y justifican la presencia de hiponatremia. La utilización de diuréticos que disminuyan la volemia puede perpetuar estos mecanismos por lo que deben evitarse ${ }^{8,9}$.

\section{Formas de presentación clínica8:}

Grado I (Hiperestimulación leve): Se caracteriza por la ausencia 
de síntomas y por un aumento del tamaño de los ovarios con presencia de múltiples quistes foliculares y cuerpos lúteos que miden hasta un máximo de $5 \times 5 \mathrm{~cm}$. Los hallazgos de laboratorio incluyen un estradiol mayor de 1500 pg/ml y niveles de progesterona mayores de $30 \mathrm{ng} / \mathrm{ml}$ en la fase inicial de la fase lútea.

Grado II (Hiperestimulación moderada): Se objetiva aumento del tamaño de los ovarios hasta $12 \times 12 \mathrm{~cm}$ acompañado de síntomas gastrointestinales tales como nauseas, vómitos, diarrea, dolor abdominal, ganancia de peso y ascitis ecográfica. Grado III (Hiperestimulación grave): Cuadro florido donde encontramos ovarios mayores de $12 \mathrm{~cm}$ y caracterizado por evidencia clínica de ascitis y/o hidrotórax y/o derrame pericárdico, hipovolemia, hemoconcentración, leucocitosis marcada, hiponatremia, oliguria, fracaso renal y disfunción hepática.

\section{Diagnóstico:}

Los clínicos que trabajen en hospitales donde se realicen técnicas de fecundación in vitro deben estar familiarizados con esta entidad. Su diagnóstico está basado una vez más en la anamnesis y la exploración física². Los estudios complementarios de imagen (ecografía) y los parámetros analíticos nos sirven para confirmar el diagnóstico y evaluar su gravedad.

\section{Manejo terapéutico:}

SHO leve: Manejo ambulatorio con reposo en cama e hidratación oral.

SHO moderado: Tratamiento sintomático con analgésicos (evitar AINEs), antieméticos, buena hidratación y reposo² ${ }^{2}$ Deben evitarse las relaciones sexuales por riesgo de ruptura de quistes y hemorragia intraabdominal ${ }^{8}$. Se debe vigilar la ganancia de peso y la diuresis diaria, realizar controles ecográficos y analíticos periódicos (hematocrito, coagulación, función renal, función hepática, proteínas e iones)². La mayoría de estos pacientes pueden manejarse de forma ambulatoria si bien la intolerancia oral o incapacidad de los pacientes para identificar signos de agravamiento constituyen razones de peso para indicar ingreso y manejo en planta de hospitalización². SHO grave: Exige ingreso en unidad de cuidados intensivos. Los pilares del tratamiento son tres. En primer lugar se debe mantener un adecuado volumen intravascular con soluciones cristaloides y expansores de plasma (albúmina humana) que asegure una buena perfusión renal ${ }^{8}$. Para ello es fundamental monitorizar la presión venosa central, el peso y las diuresis. En segundo lugar debemos realizar profilaxis de eventos tromboembólicos con $\mathrm{HBPM}^{1,2}$ y por último un manejo adecuado de complicaciones específicas del cuadro como la ascitis a tensión, el hidrotórax o el derrame pericárdico. En paciente con ascitis y/o hidrotórax la utilización de diuréticos (furosemida) es controvertida². Si bien pudiese tener algún beneficio en casos seleccionados (ausencia de hipotensión o hemoconcentración) en general parece ser un tratamiento ineficaz en disminuir el volumen acumulado en el tercer espacio a expensas de disminución del volumen intravascular con los riesgos de fracaso renal que esto implica ${ }^{2,8}$. La aspiración del líquido ascítico mediante paracentesis guiada por ecografía (riesgo de hemorragia intraperitoneal por punción de quiste ovárico a ciegas) o culdocentesis (punción y aspiración por vía transvaginal) está indicada en casos de ascitis a tensión, hidrotórax, compromiso respiratorio, oliguria, deterioro de la función renal y hemoconcentración que no responde al tratamiento médico ${ }^{2,6-8}$.

\section{Pronóstico:}

Habitualmente es bueno con resolución del cuadro en la mayoría de las pacientes aproximadamente al $7^{0}-10^{\circ}$ día. El embarazo puede constituir un factor de mal pronóstico pudiendo ser preciso interrumpirlo si la evolución no es favorable.

\section{Bibliografía}

1. Whelan JG 3rd, Vlahos NF. The ovarian hyperstimulation syndrome. Fertil Steril 2000: 73:883-96.

2. Practice Committe of the American Society for Reproductive Medicine. Ovarian hyperstimulation syndrome. Fertil Steril 2003. 80:1309-14.

3. Tang OS, Ng EH, Wai Cheng P, Chung Ho P. Cortical vein thrombosis misinterpreted as intracranial haemorrhage in severe ovarian hyperstimulation syndrome: case report. Hum Reprod 2000; 15:1913-6

4. Jing Z, Yanping L. Middle cerebral artery thrombosis after IVF and ovarian hyperstimulation: a case report. Fertil Steril 2011; 95:2435

5. Delvigne A, Rozenberg S. Epidemiology and prevention of ovarian hyperstimulation syndrome (OHSS): a review. Hum Reprod Update 2002; 8:559-77.

6. Wiser A, Levron J, Kreizer D, et al. Outcome of pregnancies complicate by severe ovarian hyperestimulation syndrome (OHSS): a follow-up beyond the second trimester. Hum Reprod 2005; 20:910-4.

7. Levin I, Almog B, Ayni A, et al. Effect of paracentesis of ascetic fluids on urinary output and blood indices in patients with severe ovarian hyperstimulation syndrome. Fertil Steril 2002; 77:986-8

8. B. Azcona, Campo G, Zabaleta J. Ovarian hyperstimulation syndrome An. Sist. Sanit. Navar. 2009; 32 (supl. 1): 19-27

9. Mathur R. Jenkins J. Ovarian hyperstimulation syndrome: an endocrinopathy? Curr Opin Obstet Gynecol. 2001; 13(3): 239-33. 